\title{
Comparison of different calcium supplementation methods in patients with osteoporosis
}

\author{
SHENG-KAI YE, XIA REN, XIANG-XUE MENG and HAI-YING CHEN \\ Department of Endocrinology, The 967th Hospital of the Chinese People's Liberation Army, \\ Dalian, Liaoning 116021, P.R. China
}

Received April 18, 2019; Accepted October 22, 2019

DOI: $10.3892 /$ etm.2019.8346

\begin{abstract}
The aim of the present study was to explore the clinical efficacy and safety of different methods of calcium supplementation in osteoporosis (OP). Patients with OP were divided into four groups, including the control, oral calcium supplementation, intravenous calcium supplementation and combined calcium supplementation groups. The duration of the calcium supplementation was 3-6 years. Medical records were collected and examined along with demographic data, the incidence of certain diseases and adverse reactions. All subjects in the three treatment groups exhibited statistically significant reductions in alkaline phosphatase (ALP) levels, visual analog score (VAS) and Oswestry Disability Index (ODI) compared with the respective pre-treatment values. Furthermore, compared with prior to treatment, the lumbar and hip bone mineral density (BMD) of patients receiving intravenous calcium supplementation was significantly increased. In addition, after treatment, the lumbar and hip BMD was significantly increased, whereas ALP levels, VAS and ODI were significantly decreased in the oral calcium supplementation group compared with the control group. Also, compared with the control and oral calcium supplementation groups, the BMD was significantly increased, whereas ALP, VAS, ODI, the rate of infection and tooth loss, the incidence of fragility fractures, joint replacement surgery, arrhythmia, lumbar disc herniation and cerebral infarction were significantly decreased in the two groups treated with intravenous calcium supplementation. The results of the present study may enable a better understanding of the effectiveness and safety of the different methods for supplementing calcium in OP.
\end{abstract}

Correspondence to: Dr Hai-Ying Chen, Department of Endocrinology, The 967th Hospital of The Chinese People's Liberation Army, 80 Shengli Road, Dalian, Liaoning 116021, P.R. China

E-mail: yeshengkai@163.com

Key words: intravenous calcium supplementation, oral calcium supplementation, osteoporosis

\section{Introduction}

Osteoporosis (OP) is a common skeletal disorder characterized by reduced bone mass and disruption of bone microarchitecture, resulting in increased bone fragility and a high fracture risk (1). The patient population suffering from OP and osteopenia is increasing annually worldwide, as is the incidence of osteoporotic fractures. It is of great concern that this condition may be encountered in any age group. Currently, the incidence of $\mathrm{OP}$ and osteoporotic fractures in adolescents is also markedly increased (2). OP is a silent disease until it is complicated by fractures, which may occur following minimal trauma or, in some cases, without trauma, and it must be viewed as part of the prevention of fractures from childhood through adulthood and old age (3). OP can be prevented, diagnosed and treated prior to the occurrence of fractures. Importantly, even after the first fracture, there are effective treatments that may be applied to decrease the risk of further fractures. Prevention, detection and treatment of OP should be mandatory among primary care providers (4).

A number of factors have been reported to be closely associated with the occurrence of OP, and they may increase the difficulty of preventing and treating OP. Moreover, a survey demonstrated that the current options for OP treatment do not appear to be promising (5), although research suggests that osteoporotic fractures are associated with a low percentage of effective OP assessment and anti-OP treatment (6). There are several available anti-OP drugs, and new drugs are continuously being developed, but several studies have consistently demonstrated that calcium supplementation can effectively improve bone mineral density (BMD), and it appears to be crucial for the prevention and treatment of OP (7). Among the currently available OP treatments, calcium is mainly administered through the oral route. There are few reports concerning the treatment of OP by intravenous calcium supplementation, and no studies have yet explored the clinical efficacy and safety of the different methods of calcium supplementation (intravenous, oral and combined) in OP.

\section{Patients and methods}

Research objectives. Between January 2011 and March 2016, 809 patients (227 male, 582 female; age range 37-89 years; mean age, $65.8 \pm 13.9$ years) with OP from the 967 th Hospital of the Chinese People's Liberation Army were included in this study 
and allocated to four groups according to the patient's wishes and initiatives, as follows: Control $(n=201)$, oral calcium supplementation $(n=198)$, intravenous calcium supplementation $(n=207)$ and combined calcium supplementation $(n=203)$ groups.

Inclusion criteria: i) OP diagnosed according to the World Health Organization (WHO) diagnostic criteria (8). ii) Informed consent to participate in this study. iii) No treatment with steroids or other drugs affecting bone metabolism, including calcium, vitamin D preparations, bisphosphonates, calcitonin (CT) and estrogen receptor modulators, for at least 3 months prior to enrolment. iv) Common diseases, including hypertension, diabetes and/or hyperlipidemia, did not affect the treatment and observation of OP. v) Study protocol approved by the Ethics Committee of the 967th Hospital of the Chinese People's Liberation Army.

Exclusion criteria: i) Psychosis or neurological dysfunction. ii) Hypercalcemia. iii) Heart failure. iv) Renal failure. v) Aberrant parathyroid hormone (PTH).

The collection, entry, follow-up and functional observation of the basic data of all patients were completed by the authors and the doctors involved in the study. In the control group, 9 cases were lost to follow-up. In the oral calcium supplementation group, 6 cases who did not comply with the prescribed treatment were excluded and 4 cases were lost to follow-up. In the intravenous calcium supplementation group, 3 cases who did not comply with the prescribed treatment were excluded and 6 cases were lost to follow-up. In the combined calcium supplementation group, 3 cases who did not comply with the prescribed treatment were excluded and 5 cases were lost to follow-up. A total of 773 patients completed the study, and their complete information was obtained. The general characteristics that were collected for the four groups of patients in this study included sex, age, body mass index (BMI), BMD, ALP, VAS, ODI, serum calcium level, prevalence of underlying conditions, smoking (Current smokers who smoked at least 7 cigarettes per week) and drinking (Drinks that contains at least 35 grams of alcohol per week) and other general conditions, and no statistically significantly differences were identified among the groups $(\mathrm{P}>0.05$; Table I).

Calcium supplementation methods. Patients in the control group received no anti-OP treatments. Patients in the oral calcium supplementation group received oral Caltrate D600 (600 mg calcium/tablet; Wyeth) daily, and patients in the intravenous calcium supplementation group were treated with a daily intravenous infusion of $10 \%$ calcium gluconate injection (10 ml, 94.7 mg calcium; Hebei Tiancheng Pharmaceutical Co., Ltd.); the course of treatment was 10 days, and patients received 1 course per quarter. In the combined calcium supplementation group, the intravenous calcium treatment protocol was the same as that for the intravenous calcium supplementation group, and the patients received oral calcium supplementation for the remaining time using the same administration protocol as described for the oral calcium supplementation group. No other anti-OP drugs were used by any of the patients. The duration of calcium supplementation was 3-6 years.

Pain and movement disorders assessment. VAS and ODI (9) were recorded prior to treatment initiation and after 3 years of treatment.
BMD assessment. Dual energy X-ray detector was used for testing. The detection sites included the lumbar vertebrae (L1-4) and the entire hip. BMD was recorded prior to treatment and after 3 years of treatment.

Intima-media thickness (IMT) assessment. The carotid IMT was detected by color Doppler ultrasound. The IMT of the posterior wall of the carotid bifurcation, common carotid and internal carotid arteries was measured, and the mean was calculated after measuring the left and right sides (10). Changes in IMT were compared before and after 3 years of treatment.

Cardiovascular system calcification assessment. Spiral computed tomography was used for the evaluation of coronary artery calcification (CAC), and the Agatston score was used for quantification (11). The total score was calculated by adding and ranking the scores of the respective lesions of the four anatomical parts (left main stem, left anterior descending branch, left circumflex branch and right coronary artery) as follows: $0-10$, no calcification; 11-100, mild calcification; 101-400, moderate calcification; 401-1,000, severe calcification; and $>1,000$, extremely severe calcification. Abdominal artery calcification (AAC) was evaluated by abdominal lateral X-ray (12). Evaluation of cardiac valve calcification was performed with echocardiography. When a strong echo of $>1 \mathrm{~mm}$ was detected on the heart valve, it was considered as valvular calcification (13) and classified as follows: Mild, single valve calcification sized 1-3 M; moderate, single valve calcification sized $3-\mathrm{mM}$, or $>2$ valvular calcifications sized 1-3 M; and severe, single valve calcification sized $>\mathrm{mM}$, or $>2$ valve calcifications sized 3-5 mm. The incidence of cardiovascular calcification was calculated in patients with new calcifications (mild) or increased degree of calcification after 3 years of treatment.

Indices and diseases monitored assessment. Indices and diseases were detected after 3 years of treatment. The criteria for the diagnosis of anomalies were as follows: Liver dysfunction (transaminase $>40.0 \mathrm{U} / 1$, normal range $0.0 \sim 40.0 \mathrm{U} / 1$ and/or total bilirubin $>25.0 \mu \mathrm{mol} / 1$, normal range $2.5 \sim 25.0 \mu \mathrm{mol} / \mathrm{l}$ ), renal dysfunction (urea $>8.0 \mathrm{mmol} / \mathrm{l}$, normal range $2.9 \sim 8.0 \mathrm{mmol} / \mathrm{l}$ and/or creatinine $>98.0 \mu \mathrm{mol} / \mathrm{l}$, normal range 57.0 98.0 $\mu \mathrm{mol} / 1)$, aberrant $\mathrm{PTH}(\leq 11.0$ and/or $\geq 67.0 \mathrm{ng} / 1$, normal range $11.0 \sim 67.0 \mathrm{ng} / \mathrm{l})$, aberrant CT ( $\geq 5.0 \mathrm{ng} / 1$, normal range $0.0 \sim 5.0 \mathrm{ng} / \mathrm{l}$ ), hypercalcemia (calcium levels were corrected for elevated albumin levels), gallstones (new cholelithiasis or increased degree) and nephrolithiasis (new nephrolithiasis or increased degree).

Statistical analysis. All statistical analyses were performed using SPSS 18.0 software (SPSS, Inc.). Student's t-test was used for enumeration data, which were expressed as mean \pm standard deviation (SD). One-way analysis of variance (ANOVA) and least significant difference post hoc tests were utilized for comparisons among multiple groups. Count data were expressed with percentages, and the Chi-square test was used for analyzing groups of count data. $\mathrm{P}<0.05$ was considered to indicate a statistically significant difference. 
Table I. Baseline characteristics of the patients in the four groups.

\begin{tabular}{lcccc}
\hline Characteristics & Control $(\mathrm{n}=192)$ & Oral calcium $(\mathrm{n}=188)$ & Intravenous calcium $(\mathrm{n}=198)$ & Combined calcium $(\mathrm{n}=195)$ \\
\hline Male, $\mathrm{n}(\%)$ & $69(35.9)$ & $62(33.0)$ & $72(36.4)$ & $67(34.4)$ \\
Age $(\mathrm{years})$ & $65.4 \pm 13.2$ & $66.2 \pm 14.1$ & $65.7 \pm 15.2$ & $65.9 \pm 13.8$ \\
BMI $\left(\mathrm{kg} / \mathrm{m}^{2}\right)$ & $22.7 \pm 2.6$ & $23.3 \pm 3.8$ & $22.9 \pm 2.4$ & $23.3 \pm 3.6$ \\
Ethnicity $[\mathrm{Han}, \mathrm{n}(\%)]$ & $173(90.1)$ & $172(91.5)$ & $181(91.4)$ & $175(89.7)$ \\
Drinking, $\mathrm{n}(\%)$ & $41(21.4)$ & $38(20.2)$ & $36(18.2)$ & $40(20.5)$ \\
Smoking, $\mathrm{n}(\%)$ & $35(18.2)$ & $32(17.0)$ & $32(16.2)$ & $34(17.4)$ \\
Hypertension, $(\%)$ & $61(31.8)$ & $60(31.9)$ & $69(34.8)$ & $66(33.8)$ \\
Diabetes, $\mathrm{n}(\%)$ & $73(38.0)$ & $69(36.7)$ & $77(38.9)$ & $74(37.9)$ \\
Hyperlipidemia, $\mathrm{n}(\%)$ & $76(39.6)$ & $73(38.8)$ & $81(40.9)$ & $76(39.0)$ \\
Serum calcium $(\mathrm{mmol} / \mathrm{l})$ & $2.36 \pm 0.24$ & $2.44 \pm 0.30$ & $2.39 \pm 0.28$ & $2.40 \pm 0.31$ \\
25(OH)D $(\mathrm{ng} / \mathrm{ml})$ & $19.63 \pm 4.29$ & $20.13 \pm 4.57$ & $19.44 \pm 5.36$ & $19.65 \pm 4.81$ \\
ALP $(\mathrm{U} / \mathrm{l})$ & $83.76 \pm 3.23$ & $84.01 \pm 3.58$ & $83.92 \pm 3.86$ & $84.33 \pm 2.87$ \\
VAS & $6.36 \pm 1.59$ & $6.34 \pm 1.77$ & $6.47 \pm 1.78$ & $6.25 \pm 1.67$ \\
ODI & $24.66 \pm 3.64$ & $24.42 \pm 3.67$ & $25.06 \pm 3.75$ & $25.12 \pm 4.29$ \\
BMDlumbar $\left(\mathrm{g} / \mathrm{cm}^{2}\right)$ & $0.764 \pm 0.134$ & $0.759 \pm 0.147$ & $0.761 \pm 0.153$ & $0.759 \pm 0.149$ \\
BMDhip $\left(\mathrm{g} / \mathrm{cm}^{2}\right)$ & $0.724 \pm 0.136$ & $0.737 \pm 0.145$ & $0.723 \pm 0.165$ & $0.720 \pm 0.141$ \\
\hline
\end{tabular}

BMI, body mass index; ALP, alkaline phosphatase; VAS, visual analog scale; ODI, Oswestry Disability Index; BMD, bone mineral density; 25(OH)D, 25-hydroxyvitamin D.

\section{Results}

Biochemical indices and the incidence of certain diseases. After treatment, no significant differences were identified among the four groups regarding the incidence of liver dysfunction, renal dysfunction, aberrant $\mathrm{PTH}$, aberrant $\mathrm{CT}$, gallstones and nephrolithiasis, coronary heart disease, heart failure and cardiovascular calcification, incidence of hypercalcemia, hypercalciuria and malignant tumors; nor were any significant differences in IMT and serum calcium levels observed (all $\mathrm{P}>0.05$; Table II).

Indices related to $O P$ and the incidence of certain diseases. All subjects in the three treatment groups (oral, intravenous and combined calcium supplementation groups) exhibited statistically significant reductions in ALP levels, VAS and ODI compared with pre-treatment values (all $\mathrm{P}<0.01$ ). Compared with prior to treatment, the BMD (lumbar and hip) of patients receiving intravenous calcium, either alone or in combination with oral calcium, was significantly increased (both $\mathrm{P}<0.01$ ). No significant differences were detected in the lumbar and hip BMD in the oral calcium supplementation group when compared before and after treatment ( $\mathrm{P}>0.05$; Table III). BMD (lumbar and hip) was significantly increased in the oral calcium supplementation group after treatment, compared with the control group $(\mathrm{P}<0.05)$, and the ALP levels, VAS and ODI were significantly decreased (all $\mathrm{P}<0.01$ ). The proportion of patients with tooth loss, infection (respiratory system, urinary system and skin infection), the incidence of fragility fractures, joint replacement surgery (knee and femoral head), arrhythmias (premature atrial contraction, tachycardia and atrial fibrillation), lumbar disc herniation (LDH) and cerebral infarction (including lacunar infarction) did not differ significantly between the oral calcium supplementation and control groups (all $\mathrm{P}>0.05$ ). In contrast to the control and oral calcium supplementation groups, the lumbar and hip BMD of patients receiving intravenous calcium treatment (intravenous and combined calcium supplementation groups) was significantly increased (both $\mathrm{P}<0.01$ ), whereas the ALP levels, VAS and ODI, the rate of infection and tooth loss, and the incidence of fragility fractures, joint replacement surgery, arrhythmias, LDH and cerebral infarction were significantly decreased [all $\mathrm{P}<0.01$, except arrhythmia $(\mathrm{P}<0.05)]$. There were no significant differences in the indices between the intravenous and combined calcium supplementation groups (all $\mathrm{P}>0.05$; Table III).

\section{Discussion}

Serum calcium is crucial for the homeostasis and normal function of multiple organ systems. Its circulating concentration is maintained within a very tight physiological range (2.25-2.75 $\mathrm{mmol} / \mathrm{l})$. Under physiological conditions, the ionized calcium concentration is regulated by PTH and CT, which are two hormones exerting opposing effects. Calcium levels in the blood are increased by PTH, while CT decreases blood calcium levels. Together, PTH and CT help regulate calcium homeostasis (14). PTH promotes the action of osteoclasts, which are cells that reabsorb bone, thus freeing up calcium. By contrast, CT decreases the activity of osteoclasts, so that calcium remains in the bone. PTH and CT accomplish their tasks through complementary pathways. Different blood concentrations of calcium trigger the secretion of PTH and CT. Low levels of blood calcium stimulate PTH secretion, and high levels of blood calcium stimulate CT secretion. Although the production and increased activity of CT is short-lived, high levels of CT oppose the effects of PTH, suppress osteoclast 
Table II. Biochemical indices and the incidence of certain diseases in the patients of the four groups after treatment.

Variables Control $(n=192)$ Oral calcium $(n=188)$ Intravenous calcium $(n=198)$ Combined $(n=195)$

\begin{tabular}{lcc}
\hline Liver dysfunction, $\mathrm{n}(\%)$ & $14(7.3)$ & $12(6.4)$ \\
Renal dysfunction, $\mathrm{n}(\%)$ & $8(4.2)$ & $9(4.8)$ \\
Gallstones, $\mathrm{n}(\%)$ & $11(5.7)$ & $12(6.4)$ \\
Nephrolithiasis, $\mathrm{n}(\%)$ & $12(6.3)$ & $11(5.9)$ \\
IMT, (mm) & $0.11 \pm 0.02$ & $0.12 \pm 0.03$ \\
Serum calcium, mmol/l & $2.36 \pm 0.24$ & $2.44 \pm 0.30$ \\
Aberrant PTH, n (\%) & $7(3.6)$ & $6(3.2)$ \\
Aberrant CT, n $(\%)$ & $6(3.1)$ & $7(3.7)$ \\
Coronary heart disease, n (\%) & $11(5.7)$ & $12(6.4)$ \\
Heart failure, n $(\%)$ & $13(6.8)$ & $13(6.9)$ \\
Cardiovascular calcification, $\mathrm{n}(\%)$ & $23(12.0)$ & $21(11.2)$ \\
Hypercalcemia, n $(\%)$ & $11(5.7)$ & $10(5.3)$ \\
Hypercalciuria, $\mathrm{n}(\%)$ & $4(2.1)$ & $3(1.6)$ \\
Malignant tumors, $\mathrm{n}(\%)$ & $8(4.2)$ & $6(3.2)$
\end{tabular}

$12(6.1)$
$8(4.0)$
$10(5.1)$
$10(5.0)$
$0.10 \pm 0.02$
$2.39 \pm 0.28$
$5(2.5)$
$7(3.5)$
$7(3.5)$
$11(5.6)$
$18(9.0)$
$9(4.5)$
$3(1.5)$
$7(3.5)$

$13(6.7)$

7 (3.6)

9 (4.6)

11 (5.6)

$0.11 \pm 0.03$

$2.40 \pm 0.31$

$6(3.1)$

5 (2.6)

8 (4.1)

$12(6.2)$

16 (8.2)

8 (4.1)

2 (1.0)

5 (2.6)

IMT, intima-media thickness; PTH, parathyroid hormone; CT, calcitonin.

Table III. Indices related to OP and the incidence of certain diseases among patients in the four groups after treatment.

\begin{tabular}{lcccc}
\hline Indices & Control $(\mathrm{n}=192)$ & Oral calcium $(\mathrm{n}=188)$ & Intravenous calcium $(\mathrm{n}=198)$ & Combined $(\mathrm{n}=195)$ \\
\hline ALP $(\mathrm{U} / \mathrm{l})$ & $87.65 \pm 4.28$ & $71.96 \pm 3.22^{\mathrm{a}-\mathrm{c}}$ & $59.17 \pm 2.35^{\mathrm{a}-\mathrm{c}}$ & $58.04 \pm 2.77^{\mathrm{a}-\mathrm{c}}$ \\
VAS & $6.19 \pm 1.53$ & $4.05 \pm 1.13^{\mathrm{a}-\mathrm{c}}$ & $1.97 \pm 0.81^{\mathrm{a}-\mathrm{c}}$ & $2.10 \pm 0.84^{\mathrm{a}-\mathrm{c}}$ \\
ODI & $26.07 \pm 3.41$ & $19.22 \pm 3.11^{\mathrm{a}-\mathrm{c}}$ & $9.61 \pm 2.17^{\mathrm{a}-\mathrm{c}}$ & $10.15 \pm 2.29^{\mathrm{a}-\mathrm{c}}$ \\
BMD lumbar $\left(\mathrm{g} / \mathrm{cm}^{2}\right)$ & $0.743 \pm 0.143$ & $0.766 \pm 0.169^{\mathrm{d}}$ & $0.833 \pm 0.177^{\mathrm{a}-\mathrm{c}}$ & $0.832 \pm 0.157^{\mathrm{a}-\mathrm{c}}$ \\
BMD hip $\left(\mathrm{g} / \mathrm{cm}^{2}\right)$ & $0.721 \pm 0.137$ & $0.739 \pm 0.172^{\mathrm{a}}$ & $0.841 \pm 0.169^{\mathrm{a}-\mathrm{c}}$ & $0.839 \pm 0.161^{\mathrm{a}-\mathrm{c}}$ \\
Fracture, $\mathrm{n}(\%)$ & $28(14.6)$ & $26(13.8)$ & $12(6.1)^{\mathrm{a}, \mathrm{b}}$ & $11(5.6)^{\mathrm{a}, \mathrm{b}}$ \\
Joint replacement, $\mathrm{n}(\%)$ & $17(8.8)$ & $16(8.5)$ & $4(2.0)^{\mathrm{a}, \mathrm{b}}$ & $5(2.6)^{\mathrm{a}, \mathrm{b}}$ \\
Tooth loss, $\mathrm{n}(\%)$ & $42(21.9)$ & $39(20.7)$ & $15(7.6)^{\mathrm{a}, \mathrm{b}}$ & $16(8.2)^{\mathrm{a}, \mathrm{b}}$ \\
LDH, $\mathrm{n}(\%)$ & $53(27.6)$ & $47(21.2)$ & $22(11.1)^{\mathrm{a}, \mathrm{b}}$ & $20(10.3)^{\mathrm{a}, \mathrm{b}}$ \\
Arrhythmias, $\mathrm{n}(\%)$ & $26(13.5)$ & $23(12.2)$ & $32(16.1)^{\mathrm{a}, \mathrm{b}}$ & $9(4.6)^{\mathrm{d}, \mathrm{e}}$ \\
Infection, $\mathrm{n}(\%)$ & $75(39.1)$ & $73(38.8)$ & $17(8.6)^{\mathrm{a}, \mathrm{b}}$ & $35(17.9)^{\mathrm{a}, \mathrm{b}}$ \\
Cerebral infarction, $\mathrm{n}(\%)$ & $37(19.3)$ & $35(18.6)$ & $18(9.2)^{\mathrm{a}, \mathrm{b}}$ \\
\hline
\end{tabular}

${ }^{a} \mathrm{P}<0.01$ vs. the control group; ${ }^{b} \mathrm{P}<0.01$ vs. the oral calcium group; ${ }^{\mathrm{c}} \mathrm{P}<0.01$ vs. pre-treatment; ${ }^{\mathrm{d}} \mathrm{P}<0.05$ vs. the control group; ${ }^{\mathrm{e}} \mathrm{P}<0.01$ vs. the oral calcium group. ALP, alkaline phosphatase; VAS, visual analog scale; ODI, Oswestry Disability Index; BMD, bone mineral density; $\mathrm{LDH}$, lumbar disk herniation.

activity and increase bone calcium (15). High levels of blood calcium may be short-lived following calcium injections. In the present study, the lumbar and hip BMD of patients treated with intravenous calcium was significantly increased compared with prior to treatment. No significant differences were identified in the lumbar and hip BMD before and after treatment in the oral calcium supplementation group. However, the lumbar and hip BMD of patients receiving intravenous calcium treatment (intravenous and combined calcium supplementation groups) was significantly increased compared with that in the control and the oral calcium supplementation groups. OP is known to be associated with calcium imbalance, although serum calcium levels are usually normal in this condition (16).
Another study indicated that there was no difference between serum calcium levels before and after calcium injections (17), which is consistent with the conclusions of the present study. The blood calcium levels in each group were within the normal range before and after treatment, and there was no significant difference among groups.

OP is characterized by skeletal fragility due to low BMD, and $\mathrm{BMD}$ is the most important factor for assessing bone strength, accounting for $\sim 70 \%$ of bone strength (18). BMD is a possible surrogate for the clinical outcome of osteoporotic fractures (19). Controlled clinical trials have demonstrated that ensuring adequate daily calcium and vitamin D intake is a safe and cost-effective method for reducing fracture 
risk (20). The present study confirmed that oral calcium and vitamin D supplementation had little effect on the prevention of osteoporotic fractures, which is consistent with the conclusions of relevant studies (21). The results of the present study demonstrated that there were no significant differences in lumbar and hip BMD before and after treatment in the oral calcium supplementation group, whereas lumbar and hip BMD was significantly increased and ALP levels, VAS and ODI were significantly decreased in the oral calcium supplementation group compared with the control group; the incidence of fragility fractures and joint replacement surgery did not differ significantly between these two groups.

The role of vitamin D in the treatment of OP is mainly to increase the intestinal absorption of calcium and phosphorus (22). Several studies suggest that various diseases, lifestyle and other factors often cause a state of vitamin D deficiency (23). Vitamin D deficiency is a global public health concern, particularly in the Chinese population (24), resulting in markedly reduced absorption of orally received calcium. A meta-analysis reported that vitamin D monotherapy had no effect on BMD (25). In the present study, the Caltrate D600 used in the oral calcium supplement group contained 125 international units of vitamin D3 per tablet, in order to compensate for vitamin D deficiency. In view of the fact that intravenous calcium supplementation directly enters into the blood circulation, no vitamin D was applied. The results of the current study suggested that lumbar and hip BMD was significantly increased in the oral calcium supplementation group after treatment, compared with the control group. In addition, the lumbar and hip BMD in the intravenous and combined calcium supplementation groups was significantly increased compared with that in the control and the oral calcium supplementation groups. These results indicate that the effect of intravenous calcium supplementation on OP is clearly superior to that of oral calcium supplementation, which may be associated with vitamin $\mathrm{D}$ to a certain extent, as vitamin $\mathrm{D}$ is not required for intravenous calcium supplementation.

Intravenous calcium has been used to treat several types of diseases, including burns, allergies (26) and cancer (27); however, the clinical efficacy of intravenous calcium supplementation for OP remains controversial to date (28-30). There have been few studies on the treatment of OP with calcium infusions in recent years, and none of those included a sufficiently large sample. To the best of our knowledge, the present study is the first to perform a detailed comparison of different calcium supplementation methods in patients with OP. The present study indicates that intravenous calcium supplementation is associated with obvious advantages in terms of the rate of infection and tooth loss, incidence of fragility fractures, joint replacement surgery, arrhythmias, LDH and cerebral infarction. In addition, intravenous calcium supplementation significantly increased BMD and improved the relevant indicators of OP. The results of the study also suggest that the clinical efficacy of intravenous calcium supplementation in OP is markedly superior to that of oral calcium supplementation, which is consistent with the findings of earlier studies $(17,31)$. The results can be interpreted to support the hypothesis that idiopathic OP results from an imbalance between PTH and CT secretion, and that calcium infusion suppresses the former and stimulates the latter. There are other possible reasons, as a number of factors are known to affect oral calcium absorption, such as calcium salt content, water solubility, the dosage and type of calcium preparation, disintegration degree, dissolution, the $\mathrm{pH}$ of the intestinal juice, the amount of gastric acid, the speed of intestinal peristalsis, plant foods or fat intake (32). It has also been suggested that, under conditions of well-balanced bone calcium metabolism, ingested calcium does not substantially enter the bone calcium pool after its absorption from the intestinal tract (33). Due to the extremely sensitive and rapid calcium balance regulation system in the human body (34), the instantaneous increase of blood calcium concentration (high levels of blood calcium may be short-lived after calcium injections) can promote the secretion of CT and suppress that of PTH, resulting in plasma calcium being transferred into the bone calcium reservoir and cells.

It was previously indicated that there is a positive correlation between OP and degeneration of the lumbar vertebrae. OP can promote the degeneration process of the lumbar vertebrae and is a high-risk factor for LDH (35). Another study indicated that LDH may cause loss of lumbar bone mass (36). In the present study, there was no statistically significant difference between the control and oral calcium supplementation groups regarding the incidence of $\mathrm{LDH}$. However, the incidence of $\mathrm{LDH}$ was significantly decreased in the groups receiving intravenous calcium supplementation compared with the control and oral calcium supplementation groups. The results of this study also suggest that $\mathrm{OP}$ and LDH are closely associated. The administration of an anti-OP treatment to patients with OP with or without $\mathrm{LDH}$, either to prevent lumbar protrusion of the intervertebral disc or to improve the symptoms, exhibits a beneficial effect.

Intracellular calcium mobilization has been implicated as an important signaling event during the internalization of several bacterial pathogens (37). Previous research also indicated that calcium plays a determinant role in the generation of pro-inflammatory responses (38). It has also been reported that calcium induces the switch from acute to chronic infection (39). OP can also increase the risk of infection (40), as it appears to be related to abnormal calcium metabolism; however, the underlying mechanism remains elusive. In this study, compared with the control group, the rate of infection (respiratory, urinary and skin infection) did not differ significantly in the oral calcium supplementation group. However, the incidence of infection in the two groups that were treated with intravenous calcium decreased significantly compared with that in the control and oral calcium supplementation groups, suggesting that anti-OP therapy with intravenous calcium supplementation may improve the body's resistance to infection.

As the pharmacological armamentarium for the management of OP has considerably expanded (41), clinicians may be faced with difficult choices when selecting the optimal treatment for each individual patient, whereas the large-scale and prolonged prescription of OP medication puts much emphasis on safety concerns. The cardiovascular safety of long-term calcium supplementation has been controversial (42), and there is increasing research focusing on their association (43). High serum calcium levels have been associated with vascular calcification (44), whereas it should be noted that a number of large-scale studies of calcium combined with vitamin D did not document an increase in cardiovascular risk $(45,46)$. The findings of the present study suggest that calcium 
supplementation as an anti-OP treatment does not increase the risk of cardiovascular calcification and other cardiovascular diseases, whereas the incidence of arrhythmias in patients receiving intravenous calcium supplements was significantly reduced. In this study, there was no significant difference in the incidence of cerebral infarction between the control and oral calcium supplementation groups, which was consistent with previous findings (47). Furthermore, the incidence of cerebral infarction in patients receiving intravenous calcium supplementation was significantly reduced.

In recent years, the possible effects of long-term calcium supplementation on the incidence of kidney stone formation have been investigated (48). According to the results of one study, calcium supplementation for the treatment of OP alone does not significantly increase the risk of nephrolithiasis (49). However, another study reported that patients with OP have a higher risk of developing gallstones compared with the general population (50). The results of the present research indicate that oral calcium and intravenous calcium supplementation did not increase the risk of cholelithiasis or nephrolithiasis during the treatment of OP.

There is significant controversy regarding the effect of calcium on the risk of cancer, with observational studies showing no effect (51), a protective effect (52), or even an increased cancer risk (53). In the present study, the incidence of malignant tumors was not significantly different among the four groups after treatment. These results indicate that neither oral nor intravenous calcium supplementation increased the risk of cancer.

The present study did not reach any further conclusions due to time and funding limitations. Further research will be focused on exploring the efficacy and safety of long-term intravenous calcium supplementation in the treatment of juvenile $\mathrm{OP}$, and the impact of intravenous calcium supplementation on the incidence of fertility disturbances and diabetes.

The incidence of OP is on the increase, and the search for effective strategies for preventing OP remains an active and challenging field of research (54). Calcium supplementation is currently the main approach to OP prevention and treatment. The results of the present study suggest that long-term oral and intravenous calcium supplementation are both effective methods for the treatment of OP, albeit the therapeutic effect of intravenous calcium supplementation is superior. Intravenous calcium may effectively improve the life quality of OP patients, and no adverse reactions were observed, which supports its application in the clinical setting.

\section{Acknowledgements}

Not applicable.

\section{Funding}

The present study was supported by the National Natural Science Foundation of China (grant no. 31730041).

\section{Availability of data and materials}

The datasets used and/or analyzed during the present study are available from the corresponding author on reasonable request.

\section{Authors' contributions}

SKY and HYC conceived and designed the study, performed the experiments, analyzed the data, prepared reviewed and edited the manuscript. XR performed the experiments and analyzed the data. XXM conceived and designed the study and revised the manuscript. All authors read and approved the manuscript.

\section{Ethics approval and consent to participate}

This study was approved by the Ethics Committee of the 967th Hospital of the Chinese People's Liberation Army (Dalian, China). Signed informed consent was obtained from the patients or their guardians.

\section{Patient consent for publication}

Not applicable.

\section{Competing interests}

The authors declare that they have no competing interests.

\section{References}

1. Ciubean AD, Ungur RA, Irsay L, Ciortea VM, Borda IM, Onac I, Vesa SC and Buzoianu AD: Health-related quality of life in Romanian postmenopausal women with osteoporosis and fragility fractures. Clin Interv Aging 13: 2465-2472, 2018.

2. Majumdar SR, Josse RG, Lin M and Eurich DT: Does sitagliptin affect the rate of osteoporotic fractures in type- 2 diabetes? Population-based cohort study. J Clin Endocrinol Metab 101: 1963-1969, 2016

3. Body JJ, Bergmann P, Boonen S, Boutsen Y, Bruyere O, Devogelaer JP, Goemaere S, Hollevoet N, Kaufman JM, Milisen K, et al: Non-pharmacological management of osteoporosis: A consensus of the Belgian Bone Club. Osteoporos Int 22: 2769-2788, 2015.

4. Cosman F, deBeur SJ, LeBoff MS, Lewiecki EM, Tanner B and Randall S: Clinician's guide to prevention and treatment of osteoporosis. Osteoporos Int 25: 2359-2381, 2014.

5. Kanis JA, McCloskey EV, Johansson H, Cooper C, Rizzoli R and Reginster JY; Scientific Advisory Board of the European Society for Clinical and Economic Aspects of Osteoporosis and Osteoarthritis (ESCEO) and the Committee of Scientific Advisors of the International Osteoporosis Foundation (IOF): European guidance for the diagnosis and management of osteoporosis in postmenopausal women. Osteoporos Int 24: 23-57, 2013.

6. Gillespie CW and Morin PE: Osteoporosis-related health services utilization following first hip fracture among a cohort of privately-insured women in the United States, 2008-2014: An Observational Study. J Bone Miner Res 32: 1052-1061, 2017.

7. Compston J, Cooper A, Cooper C, Gittoes N, Gregson C, Harvey N, Hope S, Kanis JA, McCloskey EV, Poole KES, et al: UK clinical guideline for the prevention and treatment of osteoporosis. Arch Osteoporos 12: 43, 2017.

8. Genant HK, Cooper C, Poor G, Reid I, Ehrlich G, Kanis J, Nordin BE, Barrett-Connor E, Black D, Bonjour JP, et al: Interim report and recommendations of the World Health Organization task-force for osteoporosis. Osteoporos Int 10: 259-264, 1999.

9. Grandidge L, Athanassacopolous M, Breakwell L, Chiverton N, Ivanov M and Michael R: Oswestry disability index (ODI) and visual analogue score (VAS) in pre-operative patients with radicular leg pain. Spine J 15 (Suppl): S53-S54, 2015.

10. De Pergola G, Pannacciulli N, Ciccone M, Tartagni M, Rizzon P and Giorgino R: Free testosterone plasma levels are negatively associated with the intima-media thickness of the common carotid artery in overweight and obese glucose-tolerant young adult men. Int J Obes Relat Metab Disord 27: 803-807, 2003. 
11. Ho J, FitzGerald S, Stolfus L, Cannaday J and Radford N: Severe coronary artery calcification are associated with ischemia in patients undergoing medical therapy. J Nucl Cardiol 14: 341-346, 2016.

12. Choi BH, Ro H, Jung ES, Kim AJ, Chang JH, Lee HH, Chung W and Jung JY: Circulating S100A12 levels are associated with progression of abdominal aortic calcification in hemodialysis patients. PLoS One 11: e0150145, 2016

13. Ying FT, Zhou TT, Zhou Y, Zhou YC, Liu ZH and Yu YS: The morbidity and related factors of cardiovascular calcification in peritoneal dialysis patients. J Nephrol Dialy Transplantat 24 435-440, 2015

14. Galea S and Blundell R: PTH and CT regulating calcium levels Res J Biol Sci 6: 183-186, 2011.

15. Hirsch PF, Lester GE and Talmage RV: Calcitonin, an enigmatic hormone: Does it have a function? J Musculoskelet Neuronal Interact 1: 299-305, 2001

16. Nemet AY, Hanhart J, Kaiserman I and Vinker S: Are cataracts associated with osteoporosis? Clin Ophthalmol 7: 2079-2084, 2013.

17. Hashimoto T, Matsumoto T, Awakuni J, Kosakai O, Takada H, Tsuruno S and Nakamitsu S: Hormonal change and calcium infusion therapy in osteoporosis. Orthop Traumatol 37: 333-341, 1988

18. NIH consensus development panel on osteoporosis prevention, diagnosis and therapy: Osteoporosis prevention, diagnosis, and therapy. JAMA 285: 785-795, 2001.

19. Tai V, Leung W, Grey A, Reid IR and Bolland MJ: Calcium intake and bone mineral density: Systematic review and meta-analysis. BMJ 351: h4183, 2015.

20. Larsen ER, Mosekilde L and Foldspang A: Vitamin D and calcium supplementation prevents osteoporotic fractures in elderly community dwelling residents: A pragmatic population-based 3-year intervention study. J Bone Miner Res 19: 370-378, 2004.

21. Zhao JG, Zeng XT, Wang J and Liu L: Association between Calcium or Vitamin D supplementation and fracture incidence in Community-dwelling older adults a systematic review and Meta-analysis. JAMA 318: 2466-2482, 2017.

22. Szamosi S, Horváth A, Szekanecz Z and Szúcs G: Vitamin D metabolism and osteoporosis in systemic sclerosis. Orv Hetil 158: 1252-1258, 2017 (In Hungarian).

23. Muscogiuri G, Sorice GP, Ajjan R, Mezza T, Pilz S, Prioletta A, Scragg R, Volpe SL, Witham MD and Giaccari A: Can vitamin $\mathrm{D}$ deficiency cause diabetes and cardiovascular diseases? Present evidence and future perspectives. Nutr Metab Cardiovasc Dis 22: 81-87, 2012.

24. Zhang M, Li P, Zhu Y, Chang H, Wang X, Liu W Zhang Y and Huang G: Higher visceral fat area increases the risk of vitamin $\mathrm{D}$ insufficiency and deficiency in Chinese adults. Nutr Metab (Lond) 12: 50, 2015

25. Reid IR, Bolland MJ and Grey A: Effects of vitamin D supplements on bone mineral density: A systematic review and meta-analysis. Lancet 383: 146-155, 2014.

26. Ryan JM, McCarthy G and Plunkett PK: Regional intravenous infusion of calcium Gluconate for hydrofluoric acid burns of the upper extremity. Ann Emerg Med 31: 526-527, 1998.

27. Grothey A, Nikcevich DA, Sloan JA, Kugler JW, Silberstein PT, Dentchev T Wender DB, Novotny PJ, Chitaley U, Alberts SR and Loprinzi CL: Intravenous calcium and magnesium for oxaliplatin-induced sensory neurotoxicity in adjuvant colon cancer: NCCTG N04C7. J Clin Oncol 29: 421-427, 2011

28. Jensen H, Christiansen C, Munck O and Toft H: Treatment of osteoporosis with calcium infusions an osteodensitometric study. Scand J Clin Lab Invest 32: 93-96, 1973.

29. Walton J, Dominguez M and Bartter FC: Effects of calcium infusions in patients with postmenopausal osteoporosis. Metabolism 24: 849-854, 1975.

30. Dudl RJ, Ensinck JW, Baylink D, Chesnut CH III, Sherrard D, Nelp WB and Palmieri GM: Evaluation of intravenous calcium as therapy for osteoporosis. Am J Med 55: 631-637, 1973.

31. Jowsey J, Hoye RC, Pak CY and Barter FC: The treatment of osteoporosis with calcium infusions. Evaluation of bone biopsies. Am J Med 47: 17-22, 1969.

32. Fullmer CS: Intestinal calcium absorption: Calcium entry. J Nutr 122 (3 Suppl): S644-S650, 1992.

33. Pirklbauer M, Schupart R and Mayer G: Acute calcium kinetics in hemodialysis patients. Eur J Clin Invest 46: 976-984, 2016.
34. Wyszogrodzka-Kucharska A and Rabe-Jabłońska J: Calcium balance and regulation in schizophrenic patients treated with second generation antipsychotics. Psychiatr Pol 39: 1157-1171, 2005 (In Polish)

35. Tiang P, Ma XL, Wang T, Sun XL, Fu X and Deng SC: The correlation between osteoporosis and lumbar disc herniation. J Pract Med 30: 3941-3943, 2014

36. Kim S, Jung J, Jung JH, Kim SK, Kim RB and Hahm JR: Risk factors of bone mass loss at the lumbar spine: A longitudinal study in healthy Korean Pre- and perimenopausal women older than 40 years. PLoS One 10: e0136283, 2015.

37. Asmat TM, Tenenbaum T, Jonsson AB, Schwerk $C$ and Schroten $\mathrm{H}$ : Impact of calcium signaling during infection of Neisseria meningitidis to Human Brain Microvascular endothelial cells. PLoS One 9: e114474, 2014.

38. Noble A, Truman JP, Vyas B, Vukmanovic-Steejic M, Hirst WJ and Kemeny DM: The balance of protein kinase $\mathrm{C}$ and calcium signaling directs $\mathrm{T}$ cell subset development. J Immunol 164: $1807-1813,2000$

39. Broder UN, Jaeger T and Jenal U: LadS is a calcium-responsive kinase that induces acute-to-chronic virulence switch in Pseudomonas aeruginosa. Nat Microbiol 2: 16184, 2016.

40. Xue F, Goli V, Petraro P, McMullan T, Sprafka JM and Tchetgen Tchetgen EJ: Marginal structural model to evaluate the association between cumulative osteoporosis medication and infection using claims data. Osteoporos Int 28: 2893-2901, 2017.

41. Body JJ, Bergmann P, Boonen S, Boutsen Y, Devogelaer JP, Goemaere S, Kaufman JM, Rozenberg S and Reginster JY: Evidence-based guidelines for the pharmacological treatment of postmenopausal osteoporosis: A consensus document by the Belgian Bone Club. Osteoporos Int 21: 1657-1680, 2010.

42. Chung M, Tang AM and Newberry SJ: Calcium intake and cardiovascular disease risk. Ann Intern Med 166: 686-687, 2017.

43. Tankeu AT, Ndip Agbor V and Noubiap JJ: Calcium supplementation and cardiovascular risk: A rising concern. J Clin Hypertens (Greenwich) 19: 640-646, 2017.

44. Vestergaard P, Mollerup CL, Frokjaer VG, Christiansen P, Blichert-Toft $\mathrm{M}$ and Mosekilde L: Cardiovascular events before and after surgery for primary hyperparathyroidism. World J Surg 27: 216-222, 2003

45. Jackson RD, LaCroix AZ, Gass M, Wallace RB, Robbins J, Lewis CE, Bassford T, Beresford SA, Black HR, Blanchette $\mathrm{P}$, et al: Calcium plus vitamin D supplementation and the risk of fractures. N Engl J Med 354: 669-683, 2006.

46. Hsia J, Heiss G, Ren H, Allison M, Dolan NC, Greenland P, Heckbert SR, Johnson KC, Manson JE, Sidney S, et al: Calcium/vitamin D supplementation and cardiovascular events. Circulation 115: 846-854, 2007.

47. Kong SH, Kim JH, Hong AR, Cho NH and Shin CS: Dietary calcium intake and risk of cardiovascular disease, stroke, and fracture in a population with low calcium intake. Am J Clin Nutr 106: 27-34, 2017

48. Ferraro PM, Curhan GC, D'Addessi A and Gambaro G: Risk of recurrence of idiopathic calcium kidney store: Analysis of data from the literature. J Nephrol 30: 227-233, 2017.

49. Candelas G, Martinez-Lopez JA, Rosario MP, Rosario MP, Carmona L and Loza E: Calcium supplementation and kidney stone risk in osteoporosis: A systematic literature review. Clin Exp Rheumatol 30: 954-961, 2012.

50. Klahan S, Kuo CN, Chien SC, Lin YW, Lin CY, Lin CH, Chang WC, Lin CI, Hung KS and Chang WP: Osteoporosis increases subsequent risk of gallstone: A nationwide populationbased cohort study in Taiwan. BMC Gastroenterol 14: 192, 2014

51. Park Y, Mitrou PN, Kipnis V, Hollenbeck A, Schatzkin A and Leitzmann MF: Calcium, dairy foods, and risk of incident and fatal prostate cancer: The NIH-AARP diet and health study. Am J Epidemiol 166: 1270-1279, 2007

52. Lin J, Manson JE, Lee IM, Cook NR, Buring JE and Zhang SM: Intakes of calcium and vitamin D and breast cancer risk in women. Arch Intern Med 167: 1050-1059, 2007.

53. Park Y, Leitzmann MF, Subar AF, Hollenbeck A and Schatzkin A: Dairy food, calcium, and risk of cancer in the NIH-AARP Diet and Health Study. Arch Intern Med 169: 391-401, 2009.

54. Wu X, Huang L and Liu J: Effects of adiponectin on osteoclastogenesis from mouse bone marrow-derived monocytes. Exp Ther Med 17: 1228-1233, 2019. 